\title{
HIV diagnosis period influences ART initiation: findings from a prospective cohort study in China
}

Tinglong Yang ${ }^{1+}$, Xueying Yang ${ }^{2+}$, Linghua $\mathrm{Li}^{3}$, Huifang Xu${ }^{4}$, Lirui Fan ${ }^{4}$, Quanmin $\mathrm{Li}^{3}$, Xiaoyan Fan ${ }^{1}$, Weiyi Chen ${ }^{1}$, Xuan Du' ${ }^{1}$, Chun Hao ${ }^{1,5}$, Jinghua $\mathrm{Li}^{1,5}$, Yuantao Hao ${ }^{1,5}$ and Jing Gu${ }^{1,5^{*}}$

\begin{abstract}
Background: We estimated the predictive effects of ART-related perceptions on the actual ART uptake behavior among ART naïve PLWH stratified by different time of HIV diagnosis under the new strategy.

Methods: A prospective cohort study was conducted among ART naïve PLWH in Guangzhou, China from June 2016 to June 2017. Cox regression model was used to evaluate the predictive effects of ART-related perceptions on ART initiation among PLWH stratified by different timepoint of HIV diagnosis (i.e., before or after the update of the new treatment policy).

Results: Among 411 participants, 150 and 261 were diagnosed before (pre-scaleup group) and after (post-scaleup group) the implementation of the new strategy, respectively. The ART initiation rate in the post-scaleup group (88.9\%) was higher than that in the pre-scaleup group $(73.3 \%)(p<0.001)$. A significant difference of mean score was detected in each HBM construct between pre- and post-scaleup groups $(p<0.05)$. After adjusting for significant background variables, among all participants, only the self-efficacy [adjusted $\mathrm{HR}(\mathrm{HRa})=1.23,95 \% \mathrm{Cl} 1.06$ to $1.43, p=0.006$ ], has a predictive effect on ART initiation; in pre-scaleup group, all constructs of HBM-related ART perceptions were predictors of ART initiation ( $\mathrm{HRa}=0.71$ to $1.83, p<0.05)$, while in post-scaleup group, no significant difference was found in each construct ( $p>0.05)$.

Conclusions: The ART initiation rate was high particularly among participants who diagnosed after the new treatment strategy. The important role of the time of HIV diagnosis on ART initiation identified in this study suggested that future implementation interventions may consider to modify the ART-related perceptions for HIV patients who diagnosed before the implementation of the new ART strategy, while expand the accessibility of ART service for those who diagnosed after the implementation of the new strategy.
\end{abstract}

Keywords: HIV, ART, Perceptions, Cohort, China

*Correspondence: gujing5@mail.sysu.edu.cn

${ }^{\dagger}$ Tinglong Yang and Xueying Yang contributed equally to the work

1 School of Public Health, Sun Yat-sen University, No. 74 Zhongshan Road 2, Guangzhou 510080, Guangdong, China

Full list of author information is available at the end of the article

\section{Introduction}

The HIV epidemic remained a global challenge [1]. Based on recent evidence that early antiretroviral therapy (ART) initiation was effective in preventing HIV transmission and slowing the progression of HIV-related diseases as compared to delayed treatment [2-4], WHO proposed the 'Treatment as Prevention' strategy in 2013, and recommended immediate ART for all people 
living with HIV (PLWH) irrespective of their CD4 counts (CD4) in 2015 [5]. Globally the ART coverage was 59\% in 2017 [1], and the rate varies across countries (37-72\%) [6]. In China, by the end of September 2018, the reported number of PLWH was 850,000 , and about 80,000 people are newly diagnosed every year [7]. In June 2016, China launched the new ART strategy which provides free ART irrespective of clinical stage and CD4 count and immediate initiation after diagnosis to all PLWH (hereinafter referred to as 'the New Strategy') [8].

Existing literature suggested that the factors associated with ART initiation included socio-demographic characteristics (e.g., age, education level) [9, 10], HIV infectionrelated characteristics (e.g., route of transmission, CD4 levels) $[11,12]$, perceptions about ART (e.g., knowledge, treatment intention) [13, 14] and psychosocial factors (e.g., depression, social support) [15-17]. In addition, the evolution of ART guidelines from advocating a cautious application to a near universal approach may also have crucial improvement on ART initiation. A study in Ethiopia found that PLWH who were diagnosed after year 2008 (WHO guideline increased the threshold of CD4 to initiate ART from 200 to 350 cells/ $\mu$ in 2008) were more likely to initiate ART than those diagnosed before 2008 [18]. Another study in South Africa found that among PLWH with CD4 between 201 to $350 \mathrm{cells} / \mu \mathrm{l}$, those diagnosed after 2011 (South Africa guideline increased the threshold of CD4 to start ART from 200 to 350 cells/ $\mu$ in 2011) had higher ART initiation rate than those previously diagnosed [19]. Research in China among HIV positive adolescents [20] and MSM [21] showed similar results. These cohort studies mentioned above confirmed the positive effects of broadened spectrum of eligible individuals on improving timely treatment and ART coverage.

However, there is still a significant number of PLWH who had not initiated ART despite the scale-up strategy, leading to a gap to the UNAIDS 90\% ART coverage goal [22]. How these potential ART users (e.g., past and recent diagnoses) perceive the treatment is essential to the implementation of the new strategy, as perceptions are known to be strong predictors of actual behaviors [14]. According to literature review, most existing research among ART naïve PLWH focused on the intention of early ART. For example, research in UK found that 43-47\% ART naïve PLWH were willing to start ART [23]; research in Australia found that $72 \%$ of HIV positive men who have sex with men (MSM) believed the necessity to start ART as early as possible [24]. Studies in China also reported that early ART was acceptable for over $60 \%$ of HIV positive MSM $[21,25,26]$. However, the above studies focused on ART intention and perceptions through cross-sectional designs. So far there is a lack of studies investigating how ART-related perceptions predict actual
ART initiation behaviors prospectively after the implementation of new ART strategy.

Behavioral theories help people understand, interpret and improve health-related behaviors [27, 28]. There is a number of behavioral theories being successfully applied in HIV/AIDS area, among which the Health Belief Model (HBM) is one of the most widely used. The HBM has been applied to study late ART initiation [29], ART adherence [30, 31], HIV testing [32], and was also successfully used to guide behavioral interventions [33, 34]. According to HBM, perceived severity, perceived susceptibility, perceived barriers, perceived benefits and self-efficacy are the key constructs, which can be used as potential predictors of ART initiation [27, 28]. How ART naïve PLWH perceive the treatment, whether different subgroups (e.g., PLHW diagnosed before and after the implementation of new strategy) have different perceptions, and how such differences affect ART initiation behavior? Answers to these questions are warranted when implementing the new ART strategy.

This cohort study was conducted right after the New ART strategy (universal ART) being implemented. We aimed to investigate ART-related perceptions, ART initiation behaviors and the predictive effects of perceptions on ART initiation among ART naïve PLWH in China. As the time of HIV diagnosis (before or after the implementation of the new strategy) may affect PLWH's perceptions toward ART and may lead to different behaviors, we also conducted the subgroup analyses and examined the interactions between perceptions and the time of diagnosis on ART initiation.

\section{Methods}

\section{Study design and population}

This cohort study was conducted from June 2016 to June 2018 in Guangzhou, the capital city of Guangdong Province (China started the new ART strategy since June 1st, 2016) [8]. By the end of 2015, 7311 HIV cases had been reported in Guangzhou and 5056 PLWH (69.2\%) were receiving ART (Guangzhou Yearbook, 2016). Six out of 11 districts with the highest HIV prevalence in Guangzhou were selected as the study sites, among which 3 were urban and 3 were suburb districts. All participants were recruited during the first year of the study period and followed up for at least 12 months to assess ART initiation behaviors.

The study population comprised of individuals who were aged $\geq 18$ years, confirmed as HIV positive, clinically eligible for ART, never experienced ART before, and could provide informed consent. In total, 439 eligible participants were recruited, of which 411 (93.6\%) finished the baseline survey. Ethical approval was obtained from the Ethics Committee of the School of Public Health, Sun Yat-sen University. 


\section{Data collection}

According to National Practical Guideline for the Followup and Management of PLWH, district-level Centers for Disease Control and Prevention (CDC) or Community Healthcare Centers (CHCs) (sub-district level) are responsible for providing routine care of PLWH in community, including regular follow up and health education [35]. In this study, the trained health care workers at CDC or CHCs contacted all PLWH in the jurisdiction who met the inclusion criteria and invited them to participate in the study. Potential participants were briefed about the study design, ensured the confidentiality issues, and emphasized that refusal to participate would not affect their access to any regular health care services. After informed consent, face-to-face interviews were administered in separate private rooms using structured questionnaires.

\section{Measurements}

\section{Background information}

Background information included socio-demographic characteristics (e.g., age, gender, income) and HIVrelated health status (e.g., time of HIV diagnosis, latest CD4 count) (Table 1). Participants were dichotomized by different time of HIV diagnosis into pre-scaleup group (before June 1st, 2016: the implementation of the new strategy) and post-scaleup group (after June 1st, 2016).

\section{HBM-related ART perceptions}

The HBM-based items of ART perceptions were developed through literature review, in-depth interviews with $15 \mathrm{PLWH}$, and focused group discussions. The response categories of the questions in the first four constructs were rated on a five-point Likert scale $(1=$ 'extremely disagree', $5=$ 'extremely agree').

Perceived severity was measured by four items, referring to the individual's recognition of the adverse consequences of not being treated in time (e.g., 'If you do not get treatment in time, the chance of virological failure will increase in the future'). Higher scores represented stronger belief in the serious consequences if not starting ART.

Perceived susceptibility in this study was the likelihood of transmitting HIV to one's HIV-negative sexual partner(s) and was measured by 'Non-timely participation in treatment will increase the chances of HIV transmission to sexual partners.' A higher score indicated stronger belief in the chances of HIV transmission if not starting ART.

Perceived benefits were measured by six items, referring to the individual's recognition of the benefits of timely participation in treatment (e.g., 'Starting ART may increase my CD4 counts effectively'). Higher scores indicated higher perceived benefits of starting ART.
Perceived barriers were measured by six items, referring to obstacles that an individual think they may encounter if they initiate ART (e.g., 'Early ART may lead to early side effects'). Higher scores indicated stronger belief in barriers if starting ART.

Self-efficacy was measured by five items, referring to the individual's confidence in their ability to participate in treatment successfully (e.g., 'How confident are you that you will schedule your personal and work life in order to start ART?'). Responses were measured on a five-point scale ('not', 'a little', 'not sure, 'some' and 'very'). Higher scores represented a higher level of self-efficacy to start ART immediately.

The above scales showed good internal consistency with Cronbach's alpha coefficients ranging from 0.79 to 0.92 (Table 2). For item analyses, the responses were dichotomized (e.g., agree/extremely agree, neutral/disagree/extremely disagree).

\section{Primary outcome}

The primary outcome was ART initiation, which was defined as the PLWH started ART for the first time. Their ART initiation behaviors were tracked by using the National HIV/AIDS Comprehensive Information System of China's Disease Prevention and Control information system.

\section{Statistical analysis}

In between-group comparison of the background information (participants diagnosed before or after the new strategy), $t$ test, Chi-square or rank sum test was performed accordingly. Kaplan-Meier method was used to calculate the treatment rate of the two subgroups and Log-rank test was used to test the difference.

Univariate cox regression models were fitted to assess the associations between the background variables and ART initiation in all participants. Variables with a $p<0.1$ were included as candidates in the multivariate stepwise cox regression models, and variables with a $p<0.05$ were defined as potential confounders. The associations between HBM-related ART perceptions and ART initiation was calculated by both univariate and multivariate analysis (adjusting for potential confounders). The same method was used for subgroup analysis. The results were presented as hazard ratios (HR) with 95\% confidence intervals (CI). The significance of interaction effects of time of HIV diagnosis variable on the associations between the HBM-related ART perceptions and ART initiation was tested using multiple cox regression models. A total of 5 interaction models were fitted (each construct of HBM-related ART perceptions $\times$ time of HIV diagnosis). SPSS for Windows (version 25.0; SPSS, 
Table 1 Profiles of participants diagnosed before and after implementing the new ART strategy $(n=411)$

\begin{tabular}{|c|c|c|c|c|}
\hline & All $n(\%)$ & $\begin{array}{l}\text { Diagnosed before implementing the } \\
\text { new strategy } n(\%)\end{array}$ & $\begin{array}{l}\text { Diagnosed after implementing the } \\
\text { new strategy } n(\%)\end{array}$ & $P$ \\
\hline \multicolumn{5}{|l|}{ Socio-demographic characteristics } \\
\hline Gender & & & & 0.024 \\
\hline Male & $389(94.6)$ & $137(91.3)$ & $252(96.6)$ & \\
\hline Female & $22(5.4)$ & $13(8.7)$ & $9(3.4)$ & \\
\hline Age (years, mean \pm s.d) & $32.8 \pm 10.6$ & $32.7 \pm 9.8$ & $32.9 \pm 11.1$ & 0.600 \\
\hline Age (years) & & & & 0.603 \\
\hline$\leq 25$ & $106(25.8)$ & $35(23.3)$ & $71(27.2)$ & \\
\hline $25-35$ & $172(41.8)$ & $67(44.7)$ & $105(40.2)$ & \\
\hline$>35$ & $133(32.4)$ & $48(32.0)$ & $85(32.6)$ & \\
\hline Ethnicity & & & & 0.003 \\
\hline Han & $384(93.4)$ & $133(88.7)$ & $251(96.2)$ & \\
\hline Other & $27(6.6)$ & $17(11.3)$ & $10(3.8)$ & \\
\hline Education & & & & 0.031 \\
\hline Junior high school and below & $113(27.5)$ & $50(33.3)$ & $63(24.1)$ & \\
\hline Secondary school & $128(31.1)$ & $50(33.3)$ & $78(29.9)$ & \\
\hline College or above & $170(41.4)$ & $50(33.3)$ & $120(46.0)$ & \\
\hline Monthly income (RMB) & & & & 0.522 \\
\hline$\leq 3000$ (434 USD) & $154(37.7)$ & $55(37.2)$ & $99(38.1)$ & \\
\hline 3000-5000 (434-723 USD) & $144(35.3)$ & $57(38.5)$ & $87(33.5)$ & \\
\hline > 5000 (723 USD) & $110(27.0)$ & $36(24.3)$ & $74(28.5)$ & \\
\hline Current marital status & & & & 0.384 \\
\hline Single & $255(62.0)$ & $99(66.0)$ & $156(59.8)$ & \\
\hline Married/cohabitation & $103(25.1)$ & $32(21.3)$ & $71(27.2)$ & \\
\hline Separated/divorced/other & $53(12.9)$ & $19(12.7)$ & $34(13.0)$ & \\
\hline Household registration & & & & 0.304 \\
\hline Guangzhou & $94(22.9)$ & $28(18.8)$ & $66(25.3)$ & \\
\hline Other cities of Guangdong Province & $116(28.3)$ & $43(28.9)$ & $73(28.0)$ & \\
\hline Other province & $200(48.8)$ & $78(52.3)$ & $122(46.7)$ & \\
\hline Residential district & & & & 0.384 \\
\hline Urban & $288(70.1)$ & $109(72.7)$ & $179(68.6)$ & \\
\hline Suburb & $123(29.9)$ & $41(27.3)$ & $82(31.4)$ & \\
\hline \multicolumn{5}{|l|}{ HIV infection-related conditions } \\
\hline Route of HIV infection & & & & 0.035 \\
\hline Heterosexual & $89(21.7)$ & $39(26.2)$ & $50(19.2)$ & \\
\hline Homosexual & $301(73.2)$ & $99(66.4)$ & $202(77.4)$ & \\
\hline Others (unknown, injecting drug use) & $20(4.9)$ & $11(7.4)$ & $9(3.4)$ & \\
\hline Latest CD4 cell counts & & & & $<0.001$ \\
\hline$\leq 350$ & $226(55.0)$ & $54(36.0)$ & $172(65.9)$ & \\
\hline $350-500$ & $121(29.4)$ & $60(40.0)$ & $61(23.4)$ & \\
\hline$>500$ & $64(15.6)$ & $36(24.0)$ & $28(10.7)$ & \\
\hline Self-reported health status & & & & 0.182 \\
\hline Very good/good & $168(40.9)$ & $70(46.7)$ & $98(37.5)$ & \\
\hline Not good or poor & $213(51.8)$ & $71(47.3)$ & $142(54.4)$ & \\
\hline Poor/very poor & $30(7.3)$ & $9(6.0)$ & $21(8.0)$ & \\
\hline \multicolumn{5}{|l|}{ HBM-related ART perceptions (mean \pm s.d) } \\
\hline Perceived severity & $4.1 \pm 0.7$ & $3.9 \pm 0.7$ & $4.2 \pm 0.7$ & $<0.001$ \\
\hline Perceived susceptibility & $4.3 \pm 0.8$ & $4.1 \pm 0.8$ & $4.3 \pm 0.8$ & 0.001 \\
\hline Perceived benefits & $4.2 \pm 0.6$ & $4.1 \pm 0.7$ & $4.2 \pm 0.6$ & 0.012 \\
\hline Perceived barriers & $3.1 \pm 0.7$ & $3.2 \pm 0.7$ & $3.1 \pm 0.7$ & 0.028 \\
\hline Self-efficacy & $4.2 \pm 0.8$ & $4.0 \pm 0.9$ & $4.4 \pm 0.6$ & $<0.001$ \\
\hline
\end{tabular}

Significant $P$ values are in bold 
Table 2 HBM-related ART perceptions of participants diagnosed before and after implementing the new ART strategy

\begin{tabular}{|c|c|c|c|c|}
\hline & All $n(\%)$ & $\begin{array}{l}\text { Diagnosed before } \\
\text { implementing the new } \\
\text { strategy } n(\%)\end{array}$ & $\begin{array}{l}\text { Diagnosed after } \\
\text { implementing the new } \\
\text { strategy } n(\%)\end{array}$ & $P$ \\
\hline \multicolumn{5}{|l|}{ Perceived severity ${ }^{\mathrm{a}}$ (agree/extremely agree) } \\
\hline Not starting ART may lead to poor treatment effect & $361(87.8)$ & $117(78.0)$ & $244(93.5)$ & $<0.001$ \\
\hline Not starting ART may increase the possibility of treatment failure & $344(83.7)$ & $113(75.3)$ & $231(88.5)$ & 0.001 \\
\hline Not starting ART may accelerate progress of AIDS & $370(90.0)$ & $123(82.0)$ & $247(94.6)$ & $<0.001$ \\
\hline Not starting ART may lead to drug resistance & $287(69.8)$ & $87(58.0)$ & $200(76.6)$ & $<0.001$ \\
\hline \multicolumn{5}{|l|}{ Perceived susceptibility (agree/extremely agree) } \\
\hline $\begin{array}{l}\text { Not starting ART may increase the possibility of transmitting HIV } \\
\text { to my sexual partner }\end{array}$ & $367(89.3)$ & $129(86.0)$ & $238(91.2)$ & 0.102 \\
\hline \multicolumn{5}{|l|}{ Perceived benefits ${ }^{\mathrm{b}}$ (agree/extremely agree) } \\
\hline Starting ART may increase my CD4 cell counts effectively & $372(90.5)$ & $133(88.7)$ & $239(91.6)$ & 0.333 \\
\hline $\begin{array}{l}\text { Starting ART may promote the reconstitution of my immune } \\
\text { system effectively }\end{array}$ & $364(88.6)$ & $130(86.7)$ & $234(89.7)$ & 0.359 \\
\hline Starting ART may reduce the possibility of drug resistance & $281(68.4)$ & $95(63.3)$ & $186(71.3)$ & 0.096 \\
\hline Starting ART may reduce the possibility of drug complications & $378(92.0)$ & $133(88.7)$ & $245(93.9)$ & 0.062 \\
\hline Starting ART may prolong life and reduce the possibility of death & $366(89.1)$ & $131(87.3)$ & $235(90.0)$ & 0.398 \\
\hline $\begin{array}{l}\text { Starting ART may reduce the possibility of transmitting HIV to } \\
\text { others }\end{array}$ & $342(83.2)$ & $121(80.7)$ & $221(84.7)$ & 0.295 \\
\hline \multicolumn{5}{|l|}{ Perceived barriers ${ }^{\complement}$ (agree/extremely agree) } \\
\hline Early ART may lead to early side effects & $162(39.4)$ & $61(40.7)$ & $101(38.7)$ & 0.694 \\
\hline Early ART may lead to early drug resistance & $107(26.0)$ & $38(25.3)$ & $69(26.4)$ & 0.806 \\
\hline Early ART leads to early uptake of a lifelong medication burden & $235(57.2)$ & $93(62.0)$ & $142(54.4)$ & 0.134 \\
\hline Early ART may interfere with life & $142(34.5)$ & $61(40.7)$ & $81(31.0)$ & 0.053 \\
\hline Early ART may cause an economic burden & $128(31.1)$ & $55(36.7)$ & $73(28.0)$ & 0.067 \\
\hline $\begin{array}{l}\text { Early ART may increase the possibility of disclosure of one's infec- } \\
\text { tion status }\end{array}$ & $194(47.2)$ & $84(56.0)$ & $110(42.1)$ & 0.007 \\
\hline \multicolumn{5}{|l|}{ Self-efficacy ${ }^{d}$ (some/very) } \\
\hline How confident are you that you will start ART now? & $273(66.4)$ & $90(60.0)$ & $183(70.1)$ & 0.037 \\
\hline How confident are you that you will prepare to start ART? & $331(80.5)$ & $102(71.3)$ & $124(85.8)$ & $<0.001$ \\
\hline $\begin{array}{l}\text { How confident are you that you will schedule your personal and } \\
\text { work life in order to start ART? }\end{array}$ & $312(75.9)$ & $100(66.7)$ & $212(81.2)$ & 0.001 \\
\hline $\begin{array}{l}\text { How confident are you to participate in antiviral treatment in the } \\
\text { next } 6 \text { months? }\end{array}$ & $330(80.3)$ & $101(67.3)$ & $229(87.7)$ & $<0.001$ \\
\hline $\begin{array}{l}\text { How confident are you to participate in antiviral therapy in the } \\
\text { coming year? }\end{array}$ & $341(83.0)$ & $107(71.3)$ & $234(89.7)$ & $<0.001$ \\
\hline
\end{tabular}

Significant $P$ values are in bold

${ }^{\text {a } C r o n b a c h ' s ~ a l p h a ~ c o e f f i c i e n t ~}=0.896$

${ }^{\mathrm{b}}$ Cronbach's alpha coefficient $=0.922$

${ }^{c}$ Cronbach's alpha coefficient $=0.792$

${ }^{\mathrm{d}}$ Cronbach's alpha coefficient $=0.892$

Chicago, Illinois) was used for statistical analyses. A $p$ value $<0.05$ was considered statistically significant.

\section{Results}

\section{Participants profile}

Of 411 participants, 150 (36.5\%) and 261 (63.5\%) were diagnosed before (pre-scaleup group) or after (post-scaleup group) implementing the new strategy, respectively. Most participants were male (94.6\%),
Han ethnicity (93.4\%), single (62.0\%), attained college degree or above (41.4\%), and self-report as homosexual HIV transmission route (73.2\%). The mean age was 32.8 years (standard deviation $(\mathrm{SD})=10.6$ ) and the mean value of their latest CD4 count were 347.8 cells/ $\mu \mathrm{l}(\mathrm{SD}=182.9)$. Compared with pre-scaleup group, participants in the post-scaleup group were more likely to be male ( $96.6 \%$ vs. $91.3 \%, p=0.024)$, self-report as homosexual HIV transmission route $(77.4 \%$ vs. $66.4 \%$, 
$p=0.035)$, attained college degree or above (46.0 vs. $33.3 \%, p=0.031$ ), and have a lower CD4 count (308.9 vs. $415.5, p<0.001)$ (Table 1).

\section{ART-related perception}

The difference of mean scores was significant in each construct of HBM-related ART perception between pre- and post-scaleup groups $(p<0.05)$. Specifically, the participants in the post-scaleup group perceived higher severity $(p<0.001)$, higher susceptibility $(p=0.001)$, higher benefits $(p=0.012)$, while lower perceived barriers $(p=0.028)$ and higher self-efficacy $(p<0.001)$ than those in the pre-scaleup group. Regarding specific items of HBM-related ART perception scale, $69.8 \%$ to $90.0 \%$ of all participants believed that not timely initiate ART will have serious consequences (e.g., treatment failure), especially in the post-scaleup group $(76.6 \%$ to 94.6\%), which were higher than that in the pre-scaleup group $(58.0 \%$ to $82.0 \%, p<0.05)$. Most $(89.3 \%)$ participants agreed that not timely initiate ART will increase the chance of transmitting HIV to his sexual partner(s). At the same time, most participants (68.4\% to $92.0 \%)$ perceived benefits of timely ART initiation. There was also a substantial number of participants $(26.0 \%$ to $57.2 \%$ ) who had concerns about ART (e.g., worried about the pill burden of taking ART regimen, the exposure of their HIV status, side effects). Compared with the post-scaleup group, the pre-scaleup group were more worried about the exposure of their HIV status when taking medication ( $56.0 \%$ vs. $42.1 \%, p=0.007)$. In terms of self-efficacy, $66.4 \%$ to $83.0 \%$ participants were confident to initiate ART (e.g., scheduled your personal and work life in order to start ART), and the participants in the post-scaleup group (70.1\% to $89.7 \%)$ had more confidence than those in the pre-scaleup group $(60.0 \%$ to $71.3 \%)(p<0.05)$ (Table 2$)$.

\section{ART initiation}

Among all participants, 342 (83.2\%, 95\% CI $79.6 \%$ to 86.8\%) started ART during the follow-up period. The median time interval from baseline enrollment to ART initiation of all the participants was 15 days (ranging from 1 to 758 days), with 65 (95\% CI 9.2 to 120.8) days and 12 (95\% CI 10.5 to 13.5 ) days in the pre-scaleup group and post-scaleup group, respectively. The ART initiation rate in the post-scaleup group $(88.9 \%, 95 \%$ CI $85.1 \%$ to $92.7 \%$ ) was significantly higher than that in the pre-scaleup group (73.3\%, 95\% CI $66.2 \%$ to $80.5 \%$ ) $(p<0.001)$.

\section{The predictive effect of background variables on ART initiation}

In univariate analysis of all participants, predictors of ART initiation include: older age, married/cohabited with others, living in the suburb area, higher CD4 levels, self-reported as poor health status, and diagnosed HIV after the new strategy (Table 3). In multivariate analysis (data not tabulated), participants in the post-scaleup group were more likely to initiate ART than those in the pre-scaleup group (multivariate $\mathrm{HR}\left(\mathrm{HR}_{\mathrm{m}}\right)=2.08,95 \% \mathrm{CI}$ 1.65 to $2.64, p<0.001$ ).

In univariate analysis of pre-scaleup group (Table 3), predictors of ART initiation include: older age, married/ cohabitated with others, and self-identified as homosexual transmission. In a multivariate analysis (data not tabulated), participants who were married or cohabitant with others $\left(\mathrm{HR}_{\mathrm{m}}=1.96,95 \% \mathrm{CI} 1.25\right.$ to $\left.3.07, p=0.003\right)$ were more likely to initiate ART than those who were single. In similar analysis of post-scaleup group (Table 3), participants who were living in suburban $\left(\mathrm{HR}_{\mathrm{m}}=0.73\right.$, 95\% CI 0.55 to $0.97, p=0.032$ ) and those who had more than 15 days of time interval since HIV diagnosis $\left(\mathrm{HR}_{\mathrm{m}}=0.56,95 \%\right.$ CI 0.40 to $\left.0.80, p=0.001\right)$ were less likely to initiate ART than their counterparts. There was no significant difference for latest CD4 levels in subgroup analyses between pre- and post-scaleup groups.

\section{The predictive effect of ART-related cognition variables on ART initiation}

In univariate analysis of all participants, all constructs of HBM-related ART perceptions were predictors of ART initiation $\left(\mathrm{HR}_{\mathrm{u}}=0.86\right.$ to $\left.1.38, p<0.05\right)$. After adjusting for significant background variables, only the construct of self-efficacy [adjusted $\mathrm{HR}\left(\mathrm{HR}_{\mathrm{a}}\right)=1.23,95 \%$ CI 1.06 to $1.43, p=0.006$ ] was significantly associated with ART initiation.

In univariate analyses of pre-scaleup group, participants who perceived higher severity $\left(\mathrm{HR}_{\mathrm{u}}=1.43,95 \%\right.$ CI 1.10 to $1.86, p=0.007)$, higher benefits $\left(\mathrm{HR}_{\mathrm{u}}=1.66\right.$, $95 \% \mathrm{CI} 1.23$ to $2.24, p=0.001)$, and higher selfefficacy $\left(\mathrm{HR}_{\mathrm{u}}=1.45,95 \%\right.$ CI 1.15 to $\left.1.84, p=0.002\right)$ were more likely to initiate ART, and those who perceived higher barriers $\left(\mathrm{HR}_{\mathrm{u}}=0.70,95 \% \mathrm{CI} 0.53\right.$ to 0.91 , $p=0.007)$ were less likely to initiate ART. The perceived susceptibility showed no predictive effect of ART initiation. Adjusting for significant background variables, all constructs of HBM-related ART perceptions were still predictors of ART initiation $\left(\mathrm{HR}_{\mathrm{a}}=0.71\right.$ to $\left.1.83, p<0.05\right)$ (Table 4).

In univariates and adjusted analyses of post-scaleup group, none of the constructs of HBM-related ART perceptions had predictive effect on ART initiation $(p>0.05)$ (Table 4). 


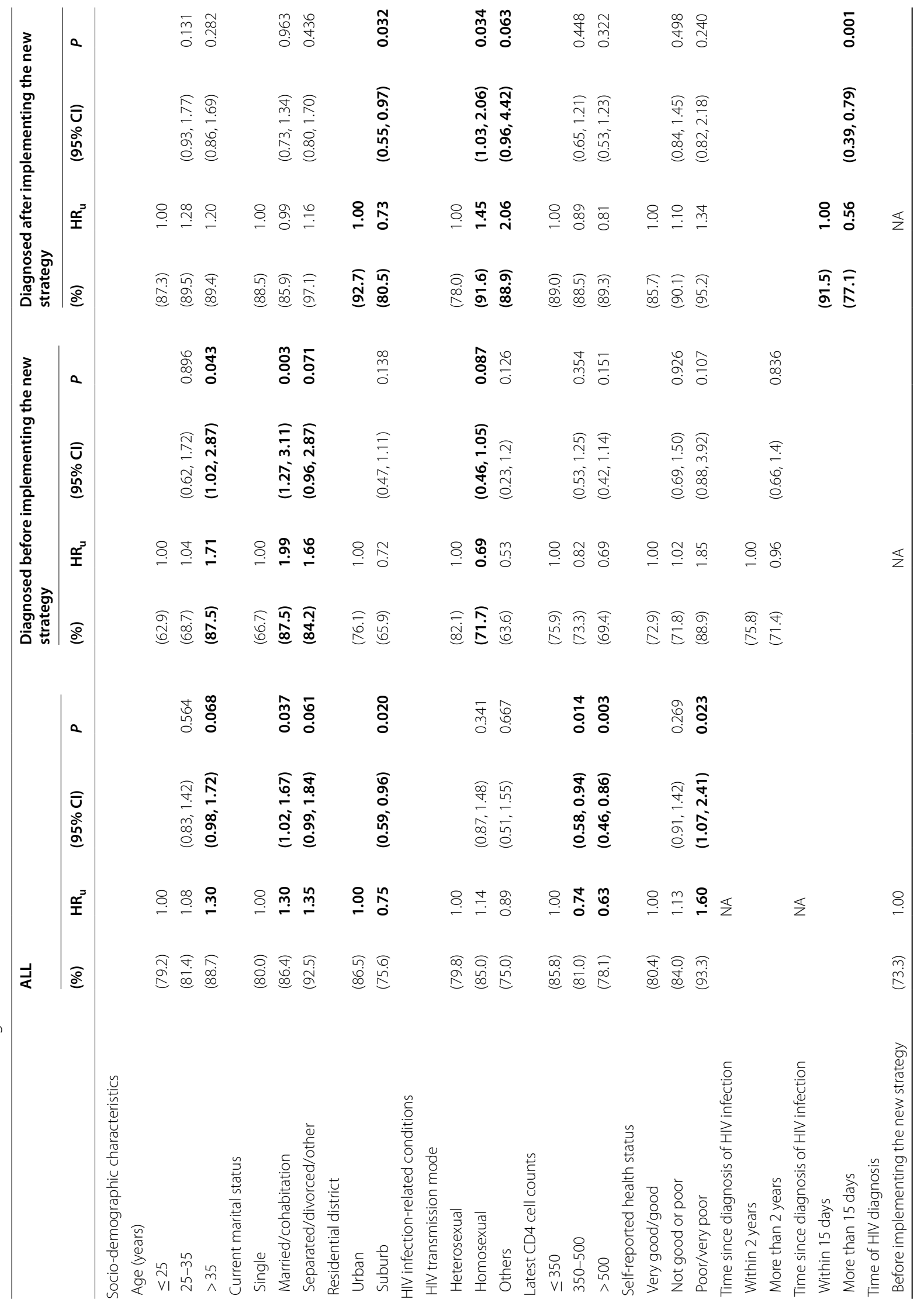




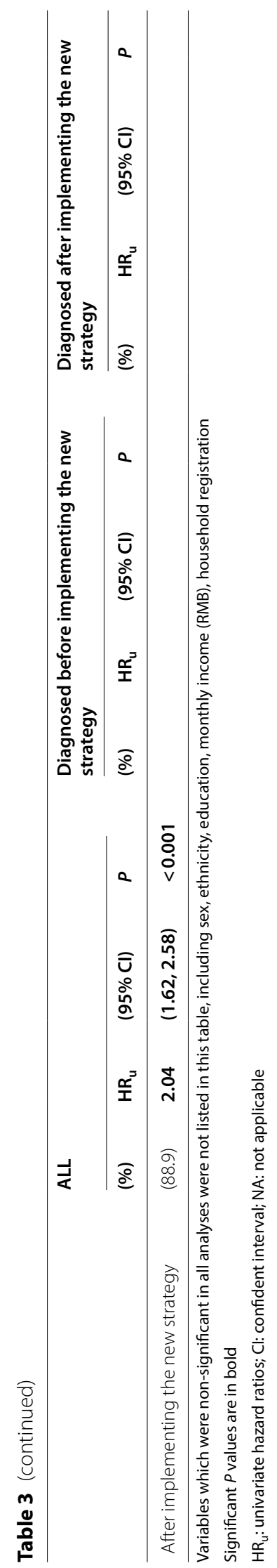




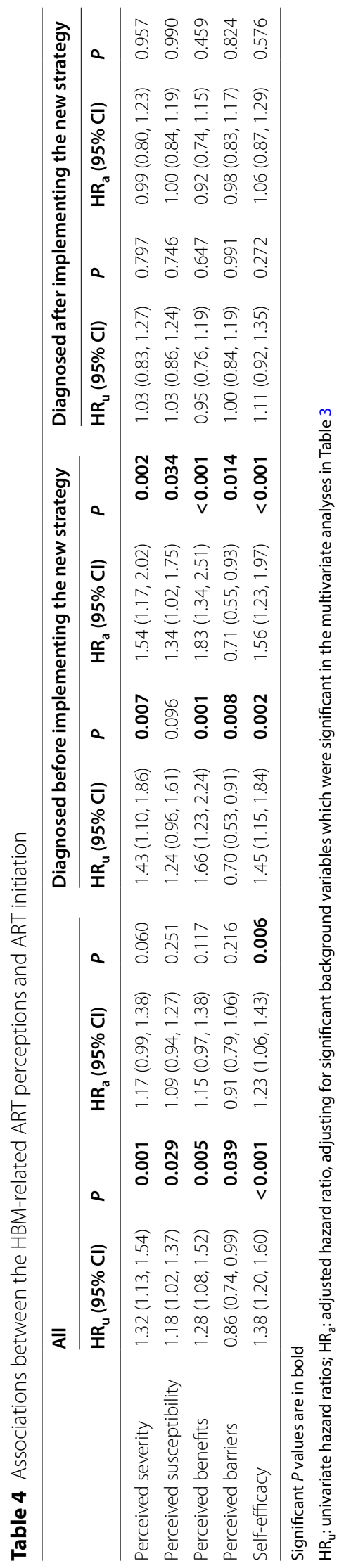




\section{Interaction}

Two out of the five models presented statistically significant interaction effect (Table 5): interaction between time of HIV diagnosis and perceived severity $(\beta=-0.36$, $p=0.038$, Fig. 1), and interaction between time of HIV diagnosis and perceived benefits $(\beta=-0.58, p=0.002$, Fig. 1). One out of the 5 models presented marginally statistically significant interaction effect [the time of HIV diagnosis and perceived barriers $(\beta=0.30, p=0.065$, Fig. 1)] (Table 5).

\section{Discussion}

This study was one of the first studies to investigate the ART initiation in a cohort among ART naïve PLWH. The results revealed that ART initiation rate was differentiated by the HIV diagnosis period and a great gap of the median time interval from enrollment to ART initiation was also identified between the two subgroups. In comparison, the ART initiation rate in the post-scaleup group is close to the $90 \%$ target, but the pre-scaleup group still has a large gap. Developing targeted interventions for ART naïve PLWH who were diagnosed before the new strategy might be beneficial for achieving the second 90 target-90\% ART coverage.

The high levels of HBM-related ART perceptions in this study may possibly be explained by the increased evidence of less side effect and more health benefits of ART $[3,4]$. We found that the participants in the post-scaleup group have more favorable ART-related perceptions than those in the pre-scaleup group in all constructs. Several reasons might account for such difference. First, the post-scaleup group received the latest and consistent ART knowledge, while the pre-scaleup group experienced multiple changes in treatment strategies, and ART knowledge obtained may be diverse and inconsistent. Second, the participants in the pre-scaleup group might still under the impression that early treatment was harmful to health and may lead to early drug resistance even if the treatment strategy changed. It may be hard to change the established perception. Third, due to the possible inadequate health promotion of the new strategies, the previously diagnosed PLWH might not comprehensively and accurately understand the new strategies. Therefore, it is necessary to conduct in-depth qualitative and quantitative research to understand the perceptions of HIV patients who diagnosed before the new strategy and to guide the future intervention designs to promote treatment.

Previous studies suggested that ART-related perceptions can be modified to promote the actual ART initiation behavior [13, 14, 29, 36-40]. The different perception levels between the two subgroups might primarily contribute to different ART initiations. Therefore, forming favorable ART-related perceptions for the pre-scaleup group may have a positive effect on ART initiation. Future research may consider using HBM for guiding intervention designs [31], such as delivering the information based on the mobile platform [41], designing short and user-friendly articles and vivid videos [42], developing a website and telephone service platform to provide more convenient consultation and support for PLWH [43], promoting advocacy in specific groups through peer support [44, 45], promoting ART initiation by supporting medical staff with knowledge, training and management skills and integrating such skills into PLWH's routine care service [46].

In addition, we found that HBM-related ART perceptions had different predictive effects on the ART initiation in different subgroups. In the pre-scaleup group, HBM-related ART perceptions have a predictive effect on the ART initiation, but in the post-scaleup group, there was no significant predictive effect. The reason may be that post-scaleup group has reached a higher level of HBM-related ART perceptions and the variation is not enough to explain the treatment behavior, and the

Table 5 Interaction between HBM-related ART perceptions and time of HIV diagnosis on ART initiation

\begin{tabular}{|c|c|c|c|}
\hline & Beta & SE (Beta) & $P$ \\
\hline \multicolumn{4}{|l|}{ Model 1} \\
\hline Perceived severity & 0.35 & 0.13 & 0.009 \\
\hline Time of HIV diagnosis ${ }^{\mathrm{a}}$ & 2.08 & 0.72 & 0.004 \\
\hline Perceived severity* time of HIV diagnosis & -0.36 & 0.17 & 0.038 \\
\hline \multicolumn{4}{|l|}{ Model 2} \\
\hline Perceived susceptibility & 0.22 & 0.13 & 0.096 \\
\hline Time of HIV diagnosis & 1.56 & 0.69 & 0.024 \\
\hline $\begin{array}{l}\text { Time of HIV diagnosis * perceived suscep- } \\
\text { tibility }\end{array}$ & -0.22 & 0.16 & 0.181 \\
\hline \multicolumn{4}{|l|}{ Model 3} \\
\hline Perceived benefits & 0.49 & 0.15 & 0.002 \\
\hline Time of HIV diagnosis & 3.06 & 0.81 & $<0.001$ \\
\hline Time of HIV diagnosis * perceived benefits & -0.58 & 0.19 & 0.002 \\
\hline \multicolumn{4}{|l|}{ Model 4} \\
\hline Perceived barriers & -0.30 & 0.14 & 0.026 \\
\hline Time of HIV diagnosis & -0.29 & 0.53 & 0.581 \\
\hline Time of HIV diagnosis * perceived barriers & 0.30 & 0.16 & 0.065 \\
\hline \multicolumn{4}{|l|}{ Model 5} \\
\hline Self-efficacy & 0.31 & 0.12 & 0.011 \\
\hline Time of HIV diagnosis & 1.34 & 0.51 & 0.008 \\
\hline Time of HIV diagnosis * self-efficacy & -0.24 & 0.16 & 0.131 \\
\hline
\end{tabular}

Each interaction model included time of HIV diagnosis, one of HBM-related ART perceptions variables and their interaction term, after adjusting for significant background variables in the multivariate analyses (District, Latest CD4 count) Significant $P$ values are in bold

${ }^{a}$ Time of HIV diagnosis has two levels, before $(=0)$ and after $(=1)$ the implementation of new ART strategy 


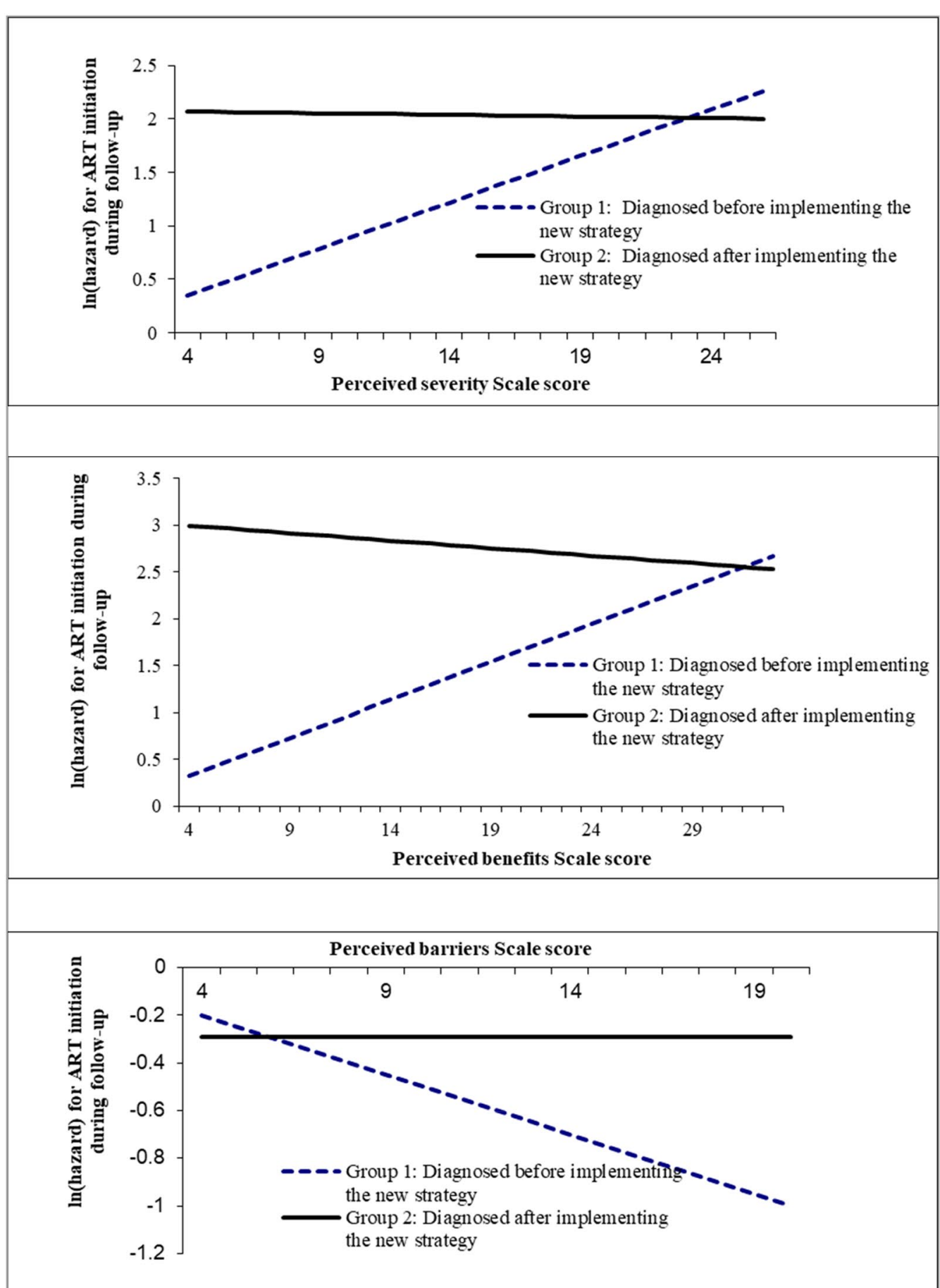

Fig. 1 Interaction effects of time of HIV diagnosis (before and after implementing the new strategy) on the associations between the HBM-related ART perceptions (perceived severity scale, perceived benefits scale and perceived barriers scale) and ART initiation

pre-scaleup group experienced multiple changes in treatment strategies and the perception variation was large $[47,48]$. Therefore, under the new strategy it may be critical to promoting the proper perceptions for the patients who diagnosed before the new ART strategy. For the post-scaleup group, implementing the immediate ART initiation program [49], increasing the accessibility of treatment could promote the ART coverage in suburban 
area, given that PLWH who were diagnosed with HIV within 15 days ( $91.5 \%$ vs. $77.1 \%$ ) and those living in urban (97.2\% vs. $80.5 \%)$ have higher treatment rate than others.

We found an interaction effect of the time of HIV diagnosis and HBM-related ART perceptions on ART initiation. According to the interaction diagram, no interaction effect of HBM-related ART perceptions and time of HIV diagnosis was found in post-scaleup group, but such interaction effect was suggested in the pre-scaleup group, which is consistent with the analyses of the effects of HBM-related ART perceptions on treatment behavior in subgroups.

In this study, we didn't find the predictive effect of CD4 levels on ART initiation among all participants and subgroup analysis. Although we observed that the higher latest CD4 levels had the lowest treatment rate of all PLWH, such association was not significant after adjusting for the time of HIV diagnosis. This result is inconsistent with previous studies which showed that higher CD4 levels was negatively associated with ART initiation [12]. The unobserved treatment disparity of different $\mathrm{CD} 4$ groups in initiating ART is quite promising.

This study has several limitations. First, we only analyzed the predictive effects of background variables and HBM-related ART perceptions on ART initiation and did not involve other factors such as psychosocial variables. Future research may consider these aspects. Second, the study defined the time to ART initiation as the interval between baseline enrollment and actual ART uptake, instead of the time interval from HIV diagnosis to ART initiation. It may not be able to directly examine the impact of new strategy on ART uptake behaviors. Third, we analyzed the ART initiation and its predictors in the two subgroups, but the imbalance in the number of cases in two subgroups may cause insufficient efficacy. Finally, the data collected by the baseline survey was selfreported data and there may be reporting bias.

\section{Conclusions}

In summary, ART initiation, HBM-related ART perceptions and predictive effect of perceptions on ART initiation were different among PLWH diagnosed at different time periods. ART coverage was high under the new strategy, especially among participants who diagnosed after the new treatment strategy. However, a gap still exists referring to the $95 \%$ treatment rate target in 2030 [50], especially in the pre-scaleup group. The important role of the time of HIV diagnosis on ART initiation identified in this study suggested that future implementation interventions may consider to modify the ART-related perceptions for HIV patients who diagnosed before the implementation of the new ART strategy, while expand the accessibility of ART service for those who diagnosed after the implementation of the new strategy.

\section{Abbreviations}

ART: Antiretroviral therapy; PLWH: People living with HIV; MSM: Men who have sex with men; UNAIDS: Joint United Nations program on AIDS/HIV; HBM: Health Belief Model; WHO: World Health Organization; CDC: Centers for Disease Control and Prevention; CHCs: Community Healthcare Centers; SD: Standard deviation; HR: Hazard ratio; $\mathrm{HR}_{\mathrm{m}}$ : Multivariate $H \mathrm{H}$; $H R_{\mathrm{u}}$ : Univariate HR; $\mathrm{HR}_{\mathrm{a}}$ : Adjusted HR.

\section{Acknowledgements}

We thank the medical staff of the Center for Disease Control and Prevention, Community Healthcare Centers (CHCs) and all participants in the study.

\section{Authors' contributions}

TY analyzed the data, edited and revised the manuscript. JG and XD conceived and designed the study and performed the research. LL, HX, LF and QL contributed to data collection and interpretation. $\mathrm{XY}$ critically reviewed the manuscript for important intellectual content and revised the manuscript. $\mathrm{CH}$, $J L, Y H, X F$ and $W C$ reviewed the manuscript. YH provided comments to the draft paper. $\mathrm{CH}, \mathrm{J}, \mathrm{YH}, \mathrm{XF}$ and WC reviewed the manuscript. All authors read and approved the final manuscript.

\section{Funding}

The study was supported by the National Natural Science Foundation Project (\#71774178), Science and Technology Planning Project of Guangzhou city (\#201607010368), Science and Technology Planning Project of Guangdong Province (\#2017A020212006), Comprehensive prevention and treatment of AIDS, viral hepatitis and tuberculosis communities Project of Guangdong Province (\#2018ZX10715004).

\section{Availability of data and materials}

The datasets used and/or analysed during the current study is available from the corresponding author on reasonable request.

\section{Declarations}

\section{Ethics approval and consent to participate}

The study protocol was reviewed and approved by the Institutional Review Board (IRB) of the School of Public Health, Sun Yat-sen University, Guangzhou, China (No: 2016-003). All subjects gave their informed consent for inclusion before they participated in the study.

\section{Consent for publication}

Not applicable.

\section{Competing interests}

YTH is a member of the editorial board of BMC Infectious Diseases and others authors have no competing interests to declare.

\section{Author details}

${ }^{1}$ School of Public Health, Sun Yat-sen University, No. 74 Zhongshan Road 2, Guangzhou 510080, Guangdong, China. ${ }^{2}$ South Carolina Smart State Center for Healthcare Quality, Department of Health Promotion, Education, and Behavior, Arnold School of Public Health, University of South Carolina, Columbia, USA. ${ }^{3}$ Department of Infectious Diseases, Guangzhou Eighth People's Hospital, Guangzhou Medical University, Guangzhou, China. ${ }^{4}$ Department of HIV Prevention, Guangzhou Center for Disease Control and Prevention, Guangzhou, China. ${ }^{5}$ Sun Yat-sen Global Health Institute, Institute of State Governance, Sun Yat-sen University, Guangzhou, China.

Received: 21 November 2020 Accepted: 11 August 2021

Published online: 09 September 2021 


\section{References}

1. World Health Organization (WHO). HIV/AIDS. Data and statistics. 2019. https://www.whoint/news-room/fact-sheets/detail/hiv-aids. Accessed 20 Oct 2019

2. Lundgren JD, Babiker AG, Gordin F, Emery S, Grund B, Sharma S, et al. Initiation of antiretroviral therapy in early asymptomatic HIV infection. N Engl J Med. 2015;373(9):795-807.

3. Danel C, Moh R, Gabillard D, Badje A, Le Carrou J, Ouassa T, et al. A trial of early antiretrovirals and isoniazid preventive therapy in Africa. N Engl J Med. 2015;373(9):808-22.

4. Grinsztejn B, Hosseinipour MC, Ribaudo HJ, Swindells S, Eron J, Chen YQ, et al. Effects of early versus delayed initiation of antiretroviral treatment on clinical outcomes of HIV-1 infection: results from the phase 3 HPTN 052 randomised controlled trial. Lancet Infect Dis. 2014;14(4):281-90.

5. World Health Organization. Guideline on when to start antiretroviral therapy and on pre-exposure prophylaxis for HIV. 2015. http://apps.who. int/iris/bitstream/10665/186275/1/9789241509565/en.pdf. Accessed 20 Oct 2019.

6. World Health Organization. 2018 Global AIDS update slides (Part 3) | UNAIDS. 2018. https://www.unaids.org/en/resources/documents/2018/ 2018-global-aids-update-slides-part3/en.pdf. Accessed 20 Oct 2019.

7. National Health Commission of the People's Republic of China. China's progress in AIDS prevention and treatment. 2018. http://www.nhc.gov. cn/zhuz/xwfb/201811/5fe377b577d04d369a057970c0f816d1.shtml. Accessed 20 Oct 2019

8. National Health Commission of the People's Republic of China. National Health and Family Planning Commission of the People's Republic of China announcement on standard of antiretroviral treatment. 2016. http://www.nhfpc.gov.cn/yzygj/s3593/201606/0b0fa78e10dc41328e84 2b1bf9cd433e.shtml. Accessed 20 Oct 2019. (in Chinese).

9. Mujugira A, Celum C, Thomas KK, Farquhar C, Mugo N, Katabira E, et al. Delay of antiretroviral therapy initiation is common in East African HIVinfected individuals in serodiscordant partnerships. J Acquir Immune Defic Syndr. 2014;66(4):436-42.

10. Mao L, de Wit J, Kippax S, Prestage G, Holt M. Younger age, recent HIV diagnosis, no welfare support and no annual sexually transmissible infection screening are associated with nonuse of antiretroviral therapy among HIV-positive gay men in Australia. HIV Med. 2015;16(1):32-7.

11. Sun J, Liu L, Shen J, Chen P, Lu H. Trends in baseline CD4 cell counts and risk factors for late antiretroviral therapy initiation among HIV-positive patients in Shanghai, a retrospective cross-sectional study. BMC Infect Dis. 2017:17(1):285.

12. Lee MJ, Venturelli S, McKenna W, Teh J, Negedu O, Florman KE, et al. Reasons for delayed antiretroviral therapy (ART) initiation in the era of early ART initiation guidelines: a retrospective service evaluation. Int J STD AIDS. 2019;30(4):415-8

13. Hollingdrake $O$, Lui CW, Mutch A, Dean J, Howard C, Fitzgerald L. Factors affecting the decision to initiate antiretroviral therapy in the era of treatment-as-prevention: synthesis of evidence from qualitative research in high-income settings. AIDS Care. 2019;31(4):397-402.

14. Ahmed S, Autrey J, Katz IT, Fox MP, Rosen S, Onoya D, et al. Why do people living with HIV not initiate treatment? A systematic review of qualitative evidence from low- and middle-income countries. Soc Sci Med. 2018;213:72-84

15. Poudel KC, Buchanan DR, Poudel-Tandukar K. Delays in antiretroviral therapy initiation among HIV-positive individuals: results of the positive living with HIV study. Glob Health Action. 2016;9:31550.

16. Velloza J, Celum C, Haberer JE, Ngure K, Irungu E, Mugo N, et al. Depression and ART initiation among HIV serodiscordant couples in Kenya and Uganda. AIDS Behav. 2017;21(8):2509-18.

17. Tao J, Vermund SH, Lu H, Ruan Y, Shepherd BE, Kipp AM, et al. Impact of depression and anxiety on initiation of antiretroviral therapy among men who have sex with men with newly diagnosed HIV infections in China. AIDS Patient Care STDS. 2017:31(2):96-104

18. Teklu AM, Delele K, Abraha M, Belayhun B, Gudina EK, Nega A. Exploratory analysis of time from HIV diagnosis to ART start, factors and effect on survival: a longitudinal follow up study at seven teaching hospitals in Ethiopia. Ethiop J Health Sci. 2017;27:17-28.

19. Plazy M, Dabis F, Naidu K, Orne-Gliemann J, Barnighausen T, Dray-Spira R. Change of treatment guidelines and evolution of ART initiation in rural
South Africa: data of a large HIV care and treatment programme. BMC Infect Dis. 2015;15:452.

20. Xu MJ, Zhao Y, Zhao DC, Ma Y. Immediate antiretrovial therapy and its predictors among HIV-infected adolescents through sexual transmission 2012-2017, China. Zhon guo ai zi bing xing bing za zhi [Chin J Prev Med]. 2019;25(04):346-9.

21. Yang $X$, Wang $Z$, Wang $X, M a T, X$ ue $H, H e Y$, et al. Behavioral intention to initiate antiretroviral therapy (ART) among Chinese HIV-infected men who have sex with men having high CD4 count in the era of "treatment for all." Am J Mens Health. 2019;13(1):1557988319828615.

22. The Joint United Nations Programme on HIV and AIDS. 90-90-90 an ambitious treatment target to help end the AIDS epidemic. Geneva SUAfhwu. https://www.unaids.org/sites/default/files/media_asset/90-9090/en.pdf. Accessed 20 Oct 2019.

23. Rodger AJ, Phillips A, Speakman A, Gilson R, Fisher M, Wilkins E, et al. Attitudes of people in the UK with HIV who are antiretroviral (ART) Naive to starting ART at high CD4 counts for potential health benefit or to prevent HIV transmission. PLoS ONE. 2014;9(5):e97340.

24. Holt M, Lea T, Murphy DA, Ellard J, Rosengarten M, Kippax SC, et al. Australian gay and bisexual men's attitudes to HIV treatment as prevention in repeated, national surveys, 2011-2013. PLoS ONE. 2014;9(11):e112349.

25. Jiang HH, Lu F, He HJ, Zhang DD, Zeng G, Xu P, et al. Acceptability status of early antiretroviral therapy among HIV-positive men who have sex with men. Zhonghua yu fang yi xue za zhi [Chin J Prev Med]. 2013;47(9):843-7.

26. Zhang Q, Tang Z, Sun H, Cheng P, Qin Q, Fan Y, et al. Acceptability of early anti-retroviral therapy among HIV-infected people in Anhui province in China. AIDS Care. 2015:27(5):669-74.

27. Albarracin D, Johnson BT, Fishbein M, Muellerleile PA. Theories of reasoned action and planned behavior as models of condom use: a metaanalysis. Psychol Bull. 2001;127(1):142-61.

28. Sheeran $\mathrm{P}$, Taylor S. Predicting intentions to use condoms: a meta-analysis and comparison of the theories of reasoned action and planned behavior 1. J Appl Soc Psychol. 2006;29:1624-75.

29. Gebru T, Lentiro K, Jemal A. Perceived behavioural predictors of late initiation to HIV/AIDS care in Gurage zone public health facilities: a cohort study using health belief model. BMC Res Notes. 2018;11(1):336.

30. Vitalis D. Predicting adherence to antiretroviral therapy among pregnant women in Guyana: utility of the health belief model. Int J STD AIDS. 2017;28(8):756-65.

31. Adefolalu AO. Cognitive-behavioural theories and adherence: application and relevance in antiretroviral therapy. S Afr J HIV Med. 2018;19(1):762.

32. Ofori KN. Application of the health belief model to HIV testing and counselling among youth living in selected rural communities in Ghana. Educ Behav Sci. 2019:5:11-8.

33. Wan LH, You LM, Chen SX, Zhang XP, Mo MM, Zhang YM, et al. The effectiveness of a comprehensive reminder system in the secondary prevention of hypertensive ischaemic stroke: randomized controlled trial protocol. J Adv Nurs. 2016;72(12):3195-206.

34. Calloway DS, Long-White DN, Corbin DE. Reducing the risk of HIV/ AIDS in African American college students: an exploratory investigation of the efficacy of a peer educator approach. Health Promot Pract. 2014;15(2):181-8

35. Huang L, Yang Q, Hu Q, Huang Q, Jiang XQ, Zhu H, et al. Analysis on the situation of the AIDS follow-up management of HIV/AIDS in Jiangxi Province. Zhou hua ji bing kong zhi za zhi [Chin J Prev Med]. 2017:21(12):1298-300.

36. Abaynew Y, Deribew A, Deribe K. Factors associated with late presentation to HIV/AIDS care in South Wollo Zone Ethiopia: a case-control study. AIDS Res Ther. 2011;8(1):8.

37. Kahn TR, Desmond M, Rao D, Marx GE, Guthrie BL, Bosire R, et al. Delayed initiation of antiretroviral therapy among HIV-discordant couples in Kenya. AIDS Care. 2013;25(3):265-72.

38. Horter S, Wringe A, Thabede Z, Dlamini V, Kerschberger B, Pasipamire $M$, et al. "Is it making any difference?" A qualitative study examining the treatment-taking experiences of asymptomatic people living with HIV in the context of treat-all in Eswatini. J Int AIDS Soc. 2019;22(1):e25220.

39. Christopoulos KA, Olender S, Lopez AM, Lekas HM, Jaiswal J, Mellman W, et al. Retained in HIV care but not on antiretroviral treatment: a qualitative patient-provider dyadic study. PLoS Med. 2015;12(8):e1001863. 
40. Persson A, Newman CE, Mao L, de Wit J. On the margins of pharmaceutical citizenship: not taking HIV medication in the "treatment revolution" era. Med Anthropol Q. 2016;30(3):359-77.

41. Venter W, Coleman J, Chan VL, Shubber Z, Phatsoane M, Gorgens M, et al. Improving linkage to HIV care through mobile phone apps: randomized controlled trial. JMIR mHealth uHealth. 2018;6(7):e155.

42. Neumann MS, Plant A, Margolis AD, Borkowf CB, Malotte CK, Rietmeijer $C A$, et al. Effects of a brief video intervention on treatment initiation and adherence among patients attending human immunodeficiency virus treatment clinics. PLoS ONE. 2018;13(10):e0204599.

43. Fox MP, Rosen S, Geldsetzer P, Barnighausen T, Negussie E, Beanland R. Interventions to improve the rate or timing of initiation of antiretroviral therapy for HIV in sub-Saharan Africa: meta-analyses of effectiveness. J Int AIDS Soc. 2016;19(1):20888.

44. Chang LW, Nakigozi G, Billioux VG, Gray RH, Serwadda D, Quinn TC, et al. Effectiveness of peer support on care engagement and preventive care intervention utilization among pre-antiretroviral therapy, HIV-infected adults in Rakai, Uganda: a randomized trial. AIDS Behav. 2015;19(10):1742-51.

45. Monroe A, Nakigozi G, Ddaaki W, Bazaale JM, Gray RH, Wawer MJ, et al. Qualitative insights into implementation, processes, and outcomes of a randomized trial on peer support and HIV care engagement in Rakai, Uganda. BMC Infect Dis. 2017;17(1):54.
46. Tembo TA, Kim MH, Simon KR, Ahmed S, Beyene T, Wetzel E, et al. Enhancing an HIV index case testing passive referral model through a behavioural skills-building training for healthcare providers: a pre-/post-assessment in Mangochi District, Malawi. J Int AIDS Soc. 2019;22(S3):e25292.

47. Down I, Prestage G, Triffitt K, Brown G, Bradley J, Ellard J. Recently diagnosed gay men talk about HIV treatment decisions. Sex Health. 2014;11(2):200-6.

48. Grace D, Chown SA, Kwag M, Steinberg M, Lim E, Gilbert M. Becoming, "undetectable": Iongitudinal narratives of gay men's sex lives after a recent HIV diagnosis. AIDS Educ Prev. 2015;27(4):333-49.

49. World Health Organization (WHO). Guidelines for managing advanced HIV disease and early initiation of antiretroviraltherapy. http://www. whoint/hiv/pub/guidelines/advanced-HIV-disease/end.pdf. Accessed 20 Oct 2019.

50. Granich R, Gupta S, Matt W, Mike R, Brian W. Modeling the HIV epidemic: why the 95-95-95 target and ART effectiveness parameters matter. Int J Virol AIDS. 2018. https://doi.org/10.23937/2469-567X/1510041.

\section{Publisher's Note}

Springer Nature remains neutral with regard to jurisdictional claims in published maps and institutional affiliations.
Ready to submit your research? Choose BMC and benefit from:

- fast, convenient online submission

- thorough peer review by experienced researchers in your field

- rapid publication on acceptance

- support for research data, including large and complex data types

- gold Open Access which fosters wider collaboration and increased citations

- maximum visibility for your research: over 100M website views per year

At BMC, research is always in progress.

Learn more biomedcentral.com/submissions 